\title{
New Analytical Method of Interdiffusion Problems
}

\author{
Hiroki Cho', Kazu-Masa Yamada², Takahisa Okino ${ }^{3 *}$ \\ ${ }^{1}$ Department of Mechanical Systems Engineering, Faculty of Environment Engineering, University of Kitakyushu, Kitakyushu, Japan \\ ${ }^{2}$ Department of Production Systems Engineering, National Institute of Technology Hakodate College, Hakodate, Japan \\ ${ }^{3}$ Applied Mathematics Department, Faculty of Science \& Engineering, Oita University, Oita, Japan \\ Email: *okino@oita-u.ac.jp
}

How to cite this paper: Cho, H., Yamada, K.-M. and Okino, T. (2018) New Analytical Method of Interdiffusion Problems. Journal of Modern Physics, 9, 130-144. https://doi.org/10.4236/jmp.2018.92009

Received: December 1, 2017

Accepted: January 16, 2018

Published: January 19, 2018

Copyright (C) 2018 by authors and Scientific Research Publishing Inc. This work is licensed under the Creative Commons Attribution International License (CC BY 4.0).

http://creativecommons.org/licenses/by/4.0/

\section{(c) (i) Open Access}

\begin{abstract}
The nonlinear diffusion equation for a binary system interdiffusion was analytically solved in the previous work. The theoretical relation of Kirkendall effect was also derived in the previous work. These new results have not yet been concretely applied to actual diffusion problems. In the present work, it is revealed that the previous results reproduce the experimental concentration profile by taking account of the movement of diffusion region space. It is thus actually confirmed that any problems of binary system interdiffusion can be solved by the new analytical method if even diffusivities of self-diffusion and impurity diffusion in the materials concerned are given. The method for solving interdiffusion problems of many elements system, which is extremely important for the development of new useful materials, is also reasonably discussed. Further, it is revealed that the concept of intrinsic diffusion is unsuitable for the diffusion theory. The fundamental theory of diffusion discussed here will be useful for analyzing actual diffusion problems in future.
\end{abstract}

\section{Keywords}

Diffusion, Many Elements System Interdiffusion, Kirkendall Effect, Parabolic Law

\section{Introduction}

The diffusion problem is one of the most fundamental and important research subjects in the material science field. The diffusion research has been thus widely and actively performed in accordance with the industry requirements for the development of new useful materials [1] [2] [3] [4] [5]. However, the progress of fundamental theory of diffusion had been hardly seen until recently. A lot of 
research papers accepting the Darken equation [6], which is mathematically wrong in the derivation process [7], have been still reported [8] [9] [10] [11] [12]. It was thus required to establish a new method for solving interdiffusion problems. In that situation, the analytical method of the nonlinear diffusion equation, which is applicable to analyzing interdiffusion problems, was reported [13] [14] [15]. Nevertheless, it does not seem that the method is really applied to analyzing results of diffusion experiments. In the present study, therefore, the analytical theory of interdiffusion problems is concretely expanded and is applied to experimental results.

First of all, we mention here fundamental concept of the diffusion theory in mathematics. In general, the usual experiments of interdiffusion between elements I and II are performed within such a temperature region that the normalized concentrations $C^{\mathrm{I}}$ and $C^{\mathrm{II}}$ satisfy the relation of

$$
C^{\mathrm{I}}+C^{\mathrm{II}}=1 \text {. }
$$

The diffusion equations for $C^{\mathrm{I}}$ and $C^{\mathrm{II}}$ in the interdiffusion field are

$$
\frac{\partial C^{\mathrm{I}}}{\partial t}=\frac{\partial}{\partial x}\left(D^{\mathrm{I}} \frac{\partial C^{\mathrm{I}}}{\partial x}\right) \text { for the element I }
$$

and

$$
\frac{\partial C^{\mathrm{II}}}{\partial t}=\frac{\partial}{\partial x}\left(D^{\mathrm{II}} \frac{\partial C^{\mathrm{II}}}{\partial x}\right) \text { for the element II, }
$$

where $D^{\mathrm{I}}$ and $D^{\mathrm{II}}$ are diffusivities for the elements I and II. Substituting Equation (1) into Equations ((2) and (3)) yields

$$
\begin{aligned}
& \frac{\partial C^{\mathrm{I}}}{\partial t}=\frac{\partial}{\partial x}\left(D^{\mathrm{I}} \frac{\partial C^{\mathrm{I}}}{\partial x}\right), \frac{\partial C^{\mathrm{II}}}{\partial t}=\frac{\partial}{\partial x}\left(D^{\mathrm{I}} \frac{\partial C^{\mathrm{II}}}{\partial x}\right) \text { and } \frac{\partial C^{\mathrm{II}}}{\partial t}=\frac{\partial}{\partial x}\left(D^{\mathrm{II}} \frac{\partial C^{\mathrm{II}}}{\partial x}\right), \\
& \frac{\partial C^{\mathrm{I}}}{\partial t}=\frac{\partial}{\partial x}\left(D^{\mathrm{II}} \frac{\partial C^{\mathrm{I}}}{\partial x}\right) .
\end{aligned}
$$

The above equations mean

$$
\left\{\frac{\partial}{\partial t}-\frac{\partial \tilde{D}}{\partial x} \frac{\partial}{\partial x}-\tilde{D} \frac{\partial^{2}}{\partial x^{2}}\right\} C^{j}=0 \text { for } j=\mathrm{I}, \mathrm{II},
$$

using the so-called interdiffusion coefficient of

$$
\tilde{D}=D^{\mathrm{I}}=D^{\mathrm{II}}
$$

Here, note that Equation (5) is valid only in the differential equation for $C^{\mathrm{I}}$ or $C^{\mathrm{II}}$ [15] [16]. The relation between diffusion fluxes $\tilde{J}^{\mathrm{I}}+\tilde{J}^{\mathrm{II}}=-\tilde{D} \partial C^{\mathrm{I}} / \partial x-$ $\tilde{D} \partial C^{\mathrm{II}} / \partial x=-\tilde{D} \partial\left(C^{\mathrm{I}}+C^{\mathrm{II}}\right) / \partial x=0$ is also valid only in the differential equation of diffusion. On the other hand, when we solve Equation (4) in accordance with the given initial and/or boundary values different from each other, the diffusivities $D^{\mathrm{I}}$ and $D^{\mathrm{II}}$ satisfying $D^{\mathrm{I}} \neq D^{\mathrm{II}}$ are naturally obtained. When we use solutions of Equations (4) for the diffusion flux $J=-D \partial C / \partial x, \quad J^{\mathrm{I}}+J^{\mathrm{II}} \neq 0$ is also valid then. There is thus no doubt that the Kirkendall effect (K effect) relevant to the essence of interdiffusion mechanism is caused by $J^{\mathrm{I}}+J^{\mathrm{II}} \neq 0 \quad$ [15] 
[16] [17] [18]. Nevertheless, the mathematical basics mentioned here had not been noticed in the long history of diffusion.

In order to understand the $\mathrm{K}$ effect, therefore, the intrinsic diffusion coefficients $D_{\text {INT }}^{\mathrm{I}}$ and $D_{\text {INT }}^{\mathrm{II}}$ satisfying $D_{\text {INT }}^{\mathrm{I}} \neq D_{\text {INT }}^{\mathrm{II}}$ were newly conceived in those days. Although the Darken equation based on the concept given by

$$
\tilde{D}=D_{\text {INT }}^{\mathrm{I}} C^{\mathrm{II}}+D_{\mathrm{INT}}^{\mathrm{II}} C^{\mathrm{I}}
$$

has been widely used for analyzing interdiffusion problems [6] [8] [9] [10] [11] [12], the equation itself is not only mathematically wrong in the derivation process but also entirely meaningless because of using the intrinsic diffusion coefficients nonexistent in the recent diffusion theory [7] [15]. In the present work, the physical meaning of $\mathrm{K}$ effect is reasonably discussed compared with experimental results, regardless of the Darken equation. As discussed at the conclusion section, the Gauss divergence theory indicates that the $\mathrm{K}$ effect may occur in the diffusion problems as universal phenomena.

Applying the analytical solutions of Equation (4) to experimental results of interdiffusion problems [17] [19] [20] [21], we confirmed that the concentration profiles are reasonably reproduced in the present work. Hereafter, as mentioned above, the Darken equation as well as the concept of intrinsic diffusion will disappear from the theory of interdiffusion problems. On the other hand, the new method discussed here will become one of the most useful methods for analyzing interdiffusion problems.

\section{Mathematical Theory of Analytical Method}

The diffusion Equations ((2) and (3)) in the time and space $(t, x)$ is generally rewritten as

$$
\frac{\partial C}{\partial t}=\frac{\partial}{\partial x}\left(D \frac{\partial C}{\partial x}\right),
$$

where the suffixes I and II are removed. When the diffusivity depends on the concentration, it had been believed until recently that the mathematical solutions of the nonlinear diffusion Equation (7) are impossible. However, the mathematical method for solving Equation (7) was established in the previous work [13] [14]. This means that the diffusion problems including a many elements system interdiffusion are essentially solved [18]. The matters necessary for the present study are briefly summarized in the following.

Boltzmann transformed Equation (7) into the ordinary differential equation of

$$
-\frac{\zeta}{2} \frac{\mathrm{d} C}{\mathrm{~d} \zeta}=\frac{\mathrm{d}}{\mathrm{d} \zeta}\left\{D \frac{\mathrm{d} C}{\mathrm{~d} \zeta}\right\},
$$

using the parabolic law $\zeta=x / \sqrt{t}$ [22]. In the previous work [13], Equation (8) was rewritten as a formula of diffusion flux in the parabolic space yielding

$$
J(\zeta)=-D(\zeta) \frac{\mathrm{d} C(\zeta)}{\mathrm{d} \zeta}
$$


where

$$
J(\zeta)=-J_{0} \exp \left[-\int_{0}^{\zeta} \frac{\eta}{2 D(\eta)} \mathrm{d} \eta\right] \text { for } J_{0}=\left.D(\zeta) \frac{\mathrm{d} C(\zeta)}{\mathrm{d} \zeta}\right|_{\zeta=0}
$$

In mathematics, the dependence of diffusivity on the concentration means

$$
\frac{\mathrm{d} C}{\mathrm{~d} \zeta}=\frac{\partial C}{\partial \zeta}+\frac{\partial C}{\partial D} \frac{\partial D}{\partial \zeta}
$$

\section{1) Impurity diffusion}

In case of the impurity diffusion, since the diffusivity of Equation (9) corresponds approximately to a constant value $D=D_{0}$, its equation becomes

$$
\frac{\mathrm{d} C(\zeta)}{\mathrm{d} \zeta}=C_{0}^{(1)} \exp \left[-\frac{\zeta^{2}}{4 D_{0}}\right],
$$

where $C_{0}^{(1)}=\mathrm{d} C /\left.\mathrm{d} \zeta\right|_{\zeta=0}$. The solution of Equation (11) is obtained as

$$
C(\zeta)=\frac{C_{\mathrm{A}}+C_{\mathrm{B}}}{2}-\frac{C_{\mathrm{A}}-C_{\mathrm{B}}}{2} \operatorname{erf}\left(\frac{\zeta}{2 \sqrt{D_{0}}}\right)
$$

for the initial condition of $C(-\infty)=C_{\mathrm{A}}$ and $C(\infty)=C_{\mathrm{B}}$ under the normalized condition of Equation (1).

\section{2) Binary system interdiffusion}

By solving simultaneously Equations ((9) and (10)), mathematical solutions of Equation ((8) or (9)) are possible (See Ref. [13]). As shown in Figure 1, under the condition of initial and/or boundary values $C_{\mathrm{A}}^{j}$, $D_{\mathrm{A}}^{j}$ of a material $\mathrm{A}$ and $C_{\mathrm{B}}^{j}, D_{\mathrm{B}}^{j}$ of a material B for $j=\mathrm{I}$, II in the binary system interdiffusion, the general solutions of Equation (9), $D^{j}(\zeta)$ and $C^{j}(\zeta)$, are obtained as

$$
D^{j}(\zeta)=\frac{D_{\mathrm{A}}^{j}+D_{\mathrm{B}}^{j}}{2}-\frac{D_{\mathrm{A}}^{j}-D_{\mathrm{B}}^{j}}{2} \operatorname{erf}\left(\frac{\zeta}{2 \sqrt{D_{\mathrm{int}}^{j}}}+\alpha^{j}\right),
$$

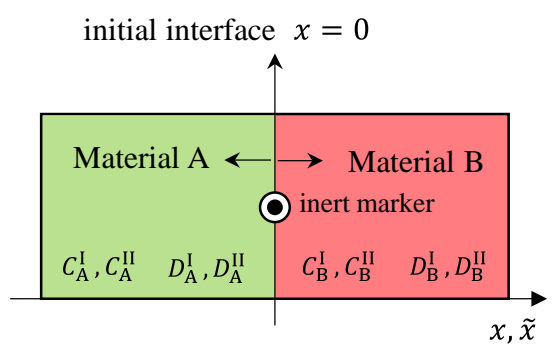

(a)

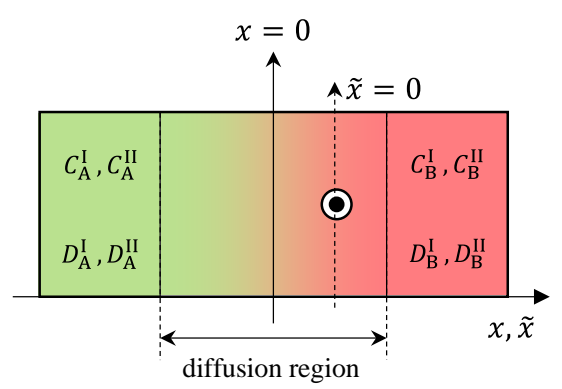

(b)

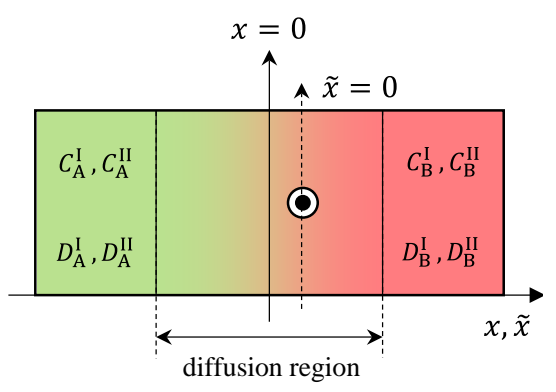

(c)

Figure 1. Schematic figure of interdiffusion phenomena. The coordinate systems of $(t, x)$ and $(\tilde{t}, \tilde{x})$ are set at a point of the mass center and at a point of space on the initial interface between materials A and B, respectively. The relation between their coordinate systems resulting from the movement of diffusion region space is conceived as $t=\tilde{t}$ and $x=\tilde{x}+\int_{0}^{t} v d \tilde{t}$ using a velocity $v$ of the origin of $(\tilde{t}, \tilde{x})$ against the origin of $(t, x)$. The notation - means an inert marker. The partial figures (a), (b) and (c) represent the initial state at a room temperature, a state at a high temperature during diffusion treatment and a state at a room temperature after diffusion treatment, respectively. 


$$
C^{j}(\zeta)=\frac{C_{\mathrm{A}}^{j}+C_{\mathrm{B}}^{j}}{2}-\frac{C_{\mathrm{A}}^{j}-C_{\mathrm{B}}^{j}}{2} \operatorname{erf}\left(\frac{\zeta}{2 \sqrt{D_{\mathrm{int}}^{j}}}+\beta^{j}\right),
$$

where $C^{\mathrm{I}}+C^{\mathrm{II}}=1, \quad D_{\text {int }}=D_{\text {int }+}^{j}=\left(D_{\mathrm{A}}^{j}+D_{\mathrm{B}}^{j}\right) / 2 \quad$ for $\quad \zeta \geq 0, \quad D_{\text {int }}=D_{\text {int- }}^{j}=$ $\sqrt{D_{\mathrm{A}}^{j} D_{\mathrm{B}}^{j}}$ for $\zeta<0$ and $\alpha^{j}=\operatorname{erf}^{-1}\left(\frac{D_{\mathrm{A}}^{j}+D_{\mathrm{B}}^{j}}{D_{\mathrm{A}}^{j}-D_{\mathrm{B}}^{j}}-\frac{2}{\ln D_{\mathrm{A}}^{j}-\ln D_{\mathrm{B}}^{j}}\right)$, $\beta^{j}=\alpha^{j}-\left(\sqrt{D_{\mathrm{A}}^{j}}-\sqrt{D_{\mathrm{B}}^{j}}\right) /\left(\sqrt{D_{\mathrm{A}}^{j}}+\sqrt{D_{\mathrm{B}}^{j}}\right)$.

We confirmed that Equations ((13) and (14)) agree well with results of the empirical Boltzmann Matano method [13] [22] [23].

In order to specify the general solutions, we must determine the initial and/or boundary values of diffusivities in the interdiffusion problem. The general method for determining them had not been experimentally and theoretically known until recently. However, they can be reasonably obtained by using the self-diffusion coefficient and impurity diffusion coefficient, since the analytical solutions are obtained. Here, note that Equations ((13) and (14)) yield the dependence of diffusivity on the concentration given by

$$
D^{j}=\frac{D_{\mathrm{A}}^{j}+D_{\mathrm{B}}^{j}}{2}-\frac{D_{\mathrm{A}}^{j}-D_{\mathrm{B}}^{j}}{2} \operatorname{erf}\left(f\left(C^{j}\right)\right)
$$

where

$$
f\left(C^{j}\right)=\operatorname{erf}^{-1}\left\{\frac{C_{\mathrm{A}}^{j}+C_{\mathrm{B}}^{j}}{C_{\mathrm{A}}^{j}-C_{\mathrm{B}}^{j}}-\frac{2 C^{j}(\zeta)}{C_{\mathrm{A}}^{j}-C_{\mathrm{B}}^{j}}\right\}+\left(\sqrt{D_{\mathrm{A}}^{j}}-\sqrt{D_{\mathrm{B}}^{j}}\right) /\left(\sqrt{D_{\mathrm{A}}^{j}}+\sqrt{D_{\mathrm{B}}^{j}}\right) .
$$

As can be easily seen, problems of binary system interdiffusion in the material composed of an arbitrary rate between elements I and II are solved by investigating the interdiffusion problem between a pure material I and a pure material II. In that case, the above initial and/or boundary values for Equations ((13) and (14)) are physically accepted as $D_{\mathrm{A}}^{\mathrm{I}}=D_{\text {self }}^{\mathrm{I}}, D_{\mathrm{B}}^{\mathrm{I}}=D_{\mathrm{imp}}^{\mathrm{I}}, C_{\mathrm{A}}^{\mathrm{I}}=1, C_{\mathrm{B}}^{\mathrm{I}}=0$, $D_{\mathrm{A}}^{\mathrm{II}}=D_{\mathrm{imp}}^{\mathrm{II}}, D_{\mathrm{B}}^{\mathrm{II}}=D_{\text {self }}^{\mathrm{II}}, C_{\mathrm{A}}^{\mathrm{II}}=0$ and $C_{\mathrm{B}}^{\mathrm{II}}=1$, using the self-diffusion coefficient $D_{\text {self }}^{j}$ of the material $j$ itself and impurity diffusion coefficient $D_{\text {imp }}^{j}$ of the material $j$ in the other material. The solutions of interdiffusion problem between pure materials I and II are thus obtained as

$$
\begin{aligned}
& D^{\mathrm{I}}(\zeta)=\frac{D_{\text {self }}^{\mathrm{I}}+D_{\text {imp }}^{\mathrm{I}}}{2}-\frac{D_{\text {self }}^{\mathrm{I}}-D_{\text {imp }}^{\mathrm{I}}}{2} \operatorname{erf}\left(\frac{\zeta}{2 \sqrt{D_{\text {int }}^{\mathrm{I}}}}+\alpha^{\mathrm{I}}\right), \\
& D^{\mathrm{II}}(\zeta)=\frac{D_{\text {imp }}^{\mathrm{II}}+D_{\text {self }}^{\mathrm{II}}}{2}-\frac{D_{\text {imp }}^{\mathrm{II}}-D_{\text {self }}^{\mathrm{II}}}{2} \operatorname{erf}\left(\frac{\zeta}{2 \sqrt{D_{\text {int }}^{\mathrm{II}}}}+\alpha^{\mathrm{II}}\right)
\end{aligned}
$$

and

$$
C^{\mathrm{I}}(\zeta)=\frac{1}{2}\left\{1-\operatorname{erf}\left(\frac{\zeta}{2 \sqrt{D_{\mathrm{int}}^{\mathrm{I}}}}+\beta^{\mathrm{I}}\right)\right\}, \quad C^{\mathrm{II}}(\zeta)=\frac{1}{2}\left\{1+\operatorname{erf}\left(\frac{\zeta}{2 \sqrt{D_{\mathrm{int}}^{\mathrm{II}}}}+\beta^{\mathrm{II}}\right)\right\} .
$$

Equations ((15a), (15b) and (15c)) show that initial and/or boundary values of diffusivities, which are applicable to any interdiffusion problems of a binary system, 
are expressed as

$$
\left\{\begin{array}{l}
D^{\mathrm{I}}=\frac{D_{\text {self }}^{\mathrm{I}}+D_{\text {imp }}^{\mathrm{I}}}{2}-\frac{D_{\text {self }}^{\mathrm{I}}-D_{\text {imp }}^{\mathrm{I}}}{2} \operatorname{erf}\left(f\left(C^{\mathrm{I}}\right)\right) \\
D^{\mathrm{II}}=\frac{D_{\text {imp }}^{\mathrm{II}}+D_{\text {self }}^{\mathrm{II}}}{2}-\frac{D_{\text {imp }}^{\mathrm{II}}-D_{\text {self }}^{\mathrm{II}}}{2} \operatorname{erf}\left(f\left(C^{\mathrm{II}}\right)\right)
\end{array}\right.
$$

where

$$
f\left(C^{j}\right)=(-1)^{j-\mathrm{I}}\left\{\operatorname{erf}^{-1}\left(1-2 C^{j}(\zeta)\right)+\left(\sqrt{D_{\text {self }}^{j}}-\sqrt{D_{\text {imp }}^{j}}\right) /\left(\sqrt{D_{\text {self }}^{j}}+\sqrt{D_{\text {imp }}^{j}}\right)\right\} .
$$

If we can experimentally obtain even diffusivities of self-diffusion and impurity diffusion in a material concerned, initial and/or boundary values necessary for solving an interdiffusion problem are thus obtained from using Equation (16).

For the interdiffusion problem of an arbitrary diffusion couple between the material A composed of $C_{\mathrm{A}}^{\mathrm{I}}=\gamma, C_{\mathrm{A}}^{\mathrm{II}}=1-\gamma$ and the material $\mathrm{B}$ composed of $C_{\mathrm{B}}^{\mathrm{I}}=\lambda, C_{\mathrm{B}}^{\mathrm{II}}=1-\lambda$, Equation (16) shows that the initial and/or boundary values of diffusivities are

$$
\left\{\begin{array}{l}
D_{\mathrm{A}}^{\mathrm{I}}=\frac{D_{\text {self }}^{\mathrm{I}}+D_{\text {imp }}^{\mathrm{I}}}{2}-\frac{D_{\text {self }}^{\mathrm{I}}-D_{\text {imp }}^{\mathrm{I}}}{2} \operatorname{erf}(f(\gamma)), \\
D_{\mathrm{B}}^{\mathrm{I}}=\frac{D_{\text {self }}^{\mathrm{I}}+D_{\mathrm{imp}}^{\mathrm{I}}}{2}-\frac{D_{\text {self }}^{\mathrm{I}}-D_{\text {imp }}^{\mathrm{I}}}{2} \operatorname{erf}(f(\lambda)) .
\end{array}\right.
$$

and

$$
\left\{\begin{array}{l}
D_{\mathrm{A}}^{\mathrm{II}}=\frac{D_{\text {imp }}^{\mathrm{II}}+D_{\text {self }}^{\mathrm{II}}}{2}-\frac{D_{\text {imp }}^{\mathrm{II}}-D_{\text {self }}^{\mathrm{II}}}{2} \operatorname{erf}(f(1-\gamma)), \\
D_{\mathrm{B}}^{\mathrm{II}}=\frac{D_{\text {imp }}^{\mathrm{II}}+D_{\text {self }}^{\mathrm{II}}}{2}-\frac{D_{\mathrm{imp}}^{\mathrm{II}}-D_{\text {self }}^{\mathrm{II}}}{2} \operatorname{erf}(f(1-\lambda)) .
\end{array}\right.
$$

The initial and/or boundary values of diffusivities corresponding to each element in a material composed of an arbitrary concentration rate for elements I and II were thus obtained as Equations ((17a) and (17b)). Therefore, the diffusivity profile of Equation (13) is obtained by using these diffusivity values of Equations $((17 a)$ and $(17 b))$. At the same time, the concentration profile of Equation (14) is also obtained by using these diffusivity values. As a matter of course, the diffusivity values of Equations $((17 \mathrm{a})$ and $(17 \mathrm{~b}))$ are also applied to $D_{\text {int }}^{j}, \alpha^{j}$ and $\beta^{j}$ in Equations ((13) and (14)).

In addition, the $\mathrm{K}$ effect shows that the diffusion region space, which is composed of vacancies and/or interstices among micro particles in a material, moves through the migration of their micro particles [17]. It is also physically considered that the diffusion region space interacts with the free space near the surface of specimen considered to be the sink and source [18]. This means that the coordinate transformation of the diffusion equation is necessary for analyzing interdiffusion problems [15] [16]. Using the solutions obtained here, the relation of $\mathrm{K}$ effect $\Delta x_{\text {eff }}$ was obtained as

$$
\Delta x_{\text {eff }}=\left|\left(\sqrt{D_{\mathrm{A}}^{\mathrm{I}}}-\sqrt{D_{\mathrm{B}}^{\mathrm{II}}}\right)\left(C_{\mathrm{A}}^{\mathrm{I}}-C_{\mathrm{B}}^{\mathrm{I}}\right)\right| \sqrt{t}
$$


in consistency with the empirical equation satisfying the parabolic law [15] [16] [18].

3) Determination of initial and/or boundary values of diffusivities in a ternary system interdiffusion

It is revealed that the so-called interdiffusion coefficient in a many elements system is meaningful only in the differential equation of diffusion [15] [18]. In the following, we first investigate the interdiffusion problem between the material $\mathrm{A}$ composed of elements I and II and the pure material B composed of an element III. Their initial concentrations are expressed as $C_{\mathrm{A}}^{\mathrm{I}}=\gamma, C_{\mathrm{A}}^{\mathrm{II}}=1-\gamma, C_{\mathrm{A}}^{\mathrm{III}}=0$, $C_{\mathrm{B}}^{\mathrm{I}}=0, C_{\mathrm{B}}^{\mathrm{II}}=0, C_{\mathrm{B}}^{\mathrm{III}}=1$ under the normalized condition of $C^{\mathrm{I}}+C^{\mathrm{II}}+C^{\mathrm{III}}=1$ in the diffusion region.

In that case, the initial and/or boundary values of diffusivities in the ternary system, $D_{\mathrm{A}}^{\mathrm{I}}=D_{\mathrm{A}, \text { ny }}^{\mathrm{I}}$ and $D_{\mathrm{A}}^{\mathrm{II}}=D_{\mathrm{A}, \text { tny }}^{\mathrm{II}}$, for I and II in the material A are considered to be $D_{\mathrm{A}, \text { tny }}^{\mathrm{I}}=D_{\mathrm{A}, \text { bny }}^{\mathrm{I}}$ and $D_{\mathrm{A} \text {, ny }}^{\mathrm{II}}=D_{\mathrm{A}, \text { bny }}^{\mathrm{II}}$, where $D^{\mathrm{I}}$ and $D^{\mathrm{II}}$ of Equations ((17a) and (17b)) obtained by analyzing the problems of binary system interdiffusion are rewritten as $D_{\mathrm{A}}^{\mathrm{I}}=D_{\mathrm{A}, \text { bny }}^{\mathrm{I}}$ and $D_{\mathrm{A}}^{\mathrm{II}}=D_{\mathrm{A}, \text { bny }}^{\mathrm{II}}$. The initial and/or boundary value of diffusivity $D_{\mathrm{A}}^{\mathrm{III}}=D_{\mathrm{A}, \text { thy }}^{\mathrm{III}}$ for III in the ternary system interdiffusion is acceptable as $D_{\mathrm{A}, \text { tny }}^{\mathrm{III}}=D_{\mathrm{A}, \mathrm{Imp}}^{\mathrm{III}}$ using the impurity diffusion coefficient $D_{\mathrm{A}, \text { imp }}^{\mathrm{III}}$ of III in the material A. In the same manner, those values $D_{\mathrm{B}, \text { tny }}^{\mathrm{I}}, D_{\mathrm{B}, \text { tny }}^{\mathrm{II}}$ and $D_{\mathrm{B}, \text { tny }}^{\mathrm{III}}$ in the material B are considered to be $D_{\mathrm{B}, \text { tny }}^{\mathrm{I}}=D_{\mathrm{B}, \text { imp }}^{\mathrm{I}}, D_{\mathrm{B}, \text { tny }}^{\mathrm{II}}=D_{\mathrm{B}, \text { imp }}^{\mathrm{II}}$ and $D_{\mathrm{B}, \text { thy }}^{\mathrm{III}}=D_{\mathrm{B}, \text { self }}^{\mathrm{III}}$, using impurity diffusion coefficients of elements I, II in the material $\mathrm{B}$ and the self-diffusion coefficient of the material B.

The present solutions of a ternary system interdiffusion problem are thus easily obtained as follows [18].

$$
\left\{\begin{array}{l}
D^{\mathrm{I}}(\zeta)=\frac{D_{\mathrm{A}, \text { bny }}^{\mathrm{I}}+D_{\mathrm{B}, \text { imp }}^{\mathrm{I}}}{2}-\frac{D_{\mathrm{A}, \text { bny }}^{\mathrm{I}}-D_{\mathrm{B}, \text { imp }}^{\mathrm{I}}}{2} \operatorname{erf}\left(\zeta / 2 \sqrt{D_{\mathrm{int}}^{\mathrm{I}}}+\alpha^{\mathrm{I}}\right) \\
D^{\mathrm{II}}(\zeta)=\frac{D_{\mathrm{A}, \text { bny }}^{\mathrm{II}}+D_{\mathrm{B}, \mathrm{imp}}^{\mathrm{II}}}{2}-\frac{D_{\mathrm{A}, \text { bny }}^{\mathrm{II}}-D_{\mathrm{B}, \mathrm{imp}}^{\mathrm{II}}}{2} \operatorname{erf}\left(\zeta / 2 \sqrt{D_{\mathrm{int}}^{\mathrm{II}}}+\alpha^{\mathrm{II}}\right) \\
D^{\mathrm{III}}(\zeta)=\frac{D_{\mathrm{A}, \mathrm{imp}}^{\mathrm{II}}+D_{\mathrm{B}, \text { self }}^{\mathrm{III}}}{2}-\frac{D_{\mathrm{A}, \mathrm{imp}}^{\mathrm{III}}-D_{\mathrm{B}, \text { self }}^{\mathrm{III}}}{2} \operatorname{erf}\left(\zeta / 2 \sqrt{D_{\mathrm{int}}^{\mathrm{III}}}+\alpha^{\mathrm{III}}\right)
\end{array}\right.
$$

where

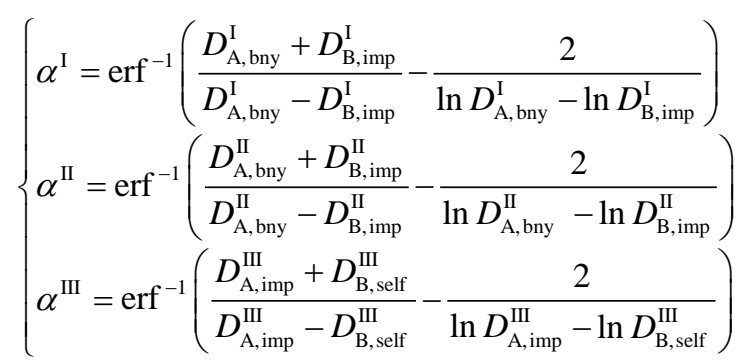

and

$$
\left\{\begin{array}{l}
D_{\mathrm{int}}^{\mathrm{I}}=D_{\mathrm{int}+}^{\mathrm{I}}=\left(D_{\mathrm{A}, \text { bny }}^{\mathrm{I}}+D_{\mathrm{B}, \text { imp }}^{\mathrm{I}}\right) / 2 \\
D_{\mathrm{int}}^{\mathrm{II}}=D_{\mathrm{int}+}^{\mathrm{II}}=\left(D_{\mathrm{A}, \text { bny }}^{\mathrm{II}}+D_{\mathrm{B}, \text { imp }}^{\mathrm{II}}\right) / 2 \text { for } \zeta \geq 0 \text { and } \\
D_{\mathrm{int}}^{\mathrm{III}}=D_{\mathrm{int}+}^{\mathrm{III}}=\left(D_{\mathrm{A}, \text { imp }}^{\mathrm{III}}+D_{\mathrm{B}, \text { self }}^{\mathrm{III}}\right) / 2
\end{array}\right.
$$


$\left\{\begin{array}{l}D_{\mathrm{int}}^{\mathrm{I}}=D_{\mathrm{int}-}^{\mathrm{I}}=\sqrt{D_{\mathrm{A}, \text { bny }}^{\mathrm{I}} D_{\mathrm{B}, \mathrm{imp}}^{\mathrm{I}}} \\ D_{\mathrm{int}}^{\mathrm{II}}=D_{\mathrm{int}-}^{\mathrm{II}}=\sqrt{D_{\mathrm{A}, \text { bny }}^{\mathrm{II}} D_{\mathrm{B}, \mathrm{imp}}^{\mathrm{II}}} \\ D_{\mathrm{int}}^{\mathrm{III}}=D_{\mathrm{int}-}^{\mathrm{III}}=\sqrt{D_{\mathrm{A}, \mathrm{imp}}^{\mathrm{III}} D_{\mathrm{B}, \text { self }}^{\mathrm{III}}}\end{array}\right.$ for $\zeta<0$.

The concentrations are

$$
\left\{\begin{array}{l}
C^{\mathrm{I}}(\zeta)=\frac{\gamma}{2}\left(1-\operatorname{erf}\left(\frac{\zeta}{2 \sqrt{D_{\mathrm{int}}^{\mathrm{I}}}}+\beta^{\mathrm{I}}\right)\right) \\
C^{\mathrm{II}}(\zeta)=\frac{1-\gamma}{2}\left(1-\operatorname{erf}\left(\frac{\zeta}{2 \sqrt{D_{\mathrm{int}}^{\mathrm{II}}}}+\beta^{\mathrm{II}}\right)\right) \\
C^{\mathrm{III}}(\zeta)=\frac{1}{2}\left(1+\operatorname{erf}\left(\frac{\zeta}{2 \sqrt{D_{\mathrm{int}}^{\mathrm{III}}}}+\beta^{\mathrm{III}}\right)\right)
\end{array}\right.
$$

where

$$
\begin{aligned}
& \beta^{\mathrm{I}}=\alpha^{\mathrm{I}}-\frac{\sqrt{D_{\mathrm{A}, \text { bny }}^{\mathrm{I}}}-\sqrt{D_{\mathrm{B}, \mathrm{imp}}^{\mathrm{I}}}}{\sqrt{D_{\mathrm{A}, \text { bny }}^{\mathrm{I}}}+\sqrt{D_{\mathrm{B}, \mathrm{imp}}^{\mathrm{I}}}}, \beta^{\mathrm{II}}=\alpha^{\mathrm{II}}-\frac{\sqrt{D_{\mathrm{A}, \text { bny }}^{\mathrm{II}}}-\sqrt{D_{\mathrm{B}, \text { imp }}^{\mathrm{II}}}}{\sqrt{D_{\mathrm{A}, \text { bny }}^{\mathrm{II}}}+\sqrt{D_{\mathrm{B}, \text { imp }}^{\mathrm{II}}}}, \\
& \beta^{\mathrm{III}}=\alpha^{\mathrm{III}}-\frac{\sqrt{D_{\mathrm{A}, \text { imp }}^{\mathrm{III}}}-\sqrt{D_{\mathrm{B}, \text { self }}^{\mathrm{III}}}}{\sqrt{D_{\mathrm{A}, \text { imp }}^{\mathrm{III}}}+\sqrt{D_{\mathrm{B}, \text { self }}^{\mathrm{II}}}} .
\end{aligned}
$$

Further, using the following relations of

$$
\left\{\begin{array}{l}
f\left(C^{\mathrm{I}}\right)=\operatorname{erf}^{-1}\left\{1-2 C^{\mathrm{I}}(\zeta) / \gamma\right\}+\left(\sqrt{D_{\mathrm{A}, \text { bny }}^{\mathrm{I}}}-\sqrt{D_{\mathrm{B}, \text { imp }}^{\mathrm{I}}}\right) /\left(\sqrt{D_{\mathrm{A}, \text { bny }}^{\mathrm{I}}}+\sqrt{D_{\mathrm{B}, \text { imp }}^{\mathrm{I}}}\right) \\
f\left(C^{\mathrm{II}}\right)=\operatorname{erf}^{-1}\left\{1-2 C^{\mathrm{II}}(\zeta) /(1-\gamma)\right\}+\left(\sqrt{D_{\mathrm{A}, \text { bny }}^{\mathrm{II}}}-\sqrt{D_{\mathrm{B}, \text { imp }}^{\mathrm{II}}}\right) /\left(\sqrt{D_{\mathrm{A}, \text { bny }}^{\mathrm{II}}}+\sqrt{D_{\mathrm{B}, \text { imp }}^{\mathrm{II}}}\right) \\
f\left(C^{\mathrm{III}}\right)=\operatorname{erf}^{-1}\left\{2 C^{\mathrm{III}}(\zeta)-1\right\}+\left(\sqrt{D_{\mathrm{A}, \text { imp }}^{\mathrm{III}}}-\sqrt{D_{\mathrm{B}, \text { self }}^{\mathrm{III}}}\right) /\left(\sqrt{D_{\mathrm{A}, \text { imp }}^{\mathrm{III}}}+\sqrt{D_{\mathrm{B}, \text { self }}^{\mathrm{III}}}\right)
\end{array}\right.
$$

the dependences of diffusivity on the concentration are obtained as

$$
\left\{\begin{array}{l}
D^{\mathrm{I}}(\zeta)=\frac{D_{\mathrm{A}, \text { bny }}^{\mathrm{I}}+D_{\mathrm{B}, \text { imp }}^{\mathrm{I}}}{2}-\frac{D_{\mathrm{A}, \text { bny }}^{\mathrm{I}}-D_{\mathrm{B}, \text { imp }}^{\mathrm{I}}}{2} \operatorname{erf}\left(f\left(C^{\mathrm{I}}\right)\right) \\
D^{\mathrm{II}}(\zeta)=\frac{D_{\mathrm{A}, \text { bny }}^{\mathrm{II}}+D_{\mathrm{B}, \text { imp }}^{\mathrm{II}}}{2}-\frac{D_{\mathrm{A}, \text { bny }}^{\mathrm{II}}-D_{\mathrm{B}, \text { imp }}^{\mathrm{II}}}{2} \operatorname{erf}\left(f\left(C^{\mathrm{II}}\right)\right) . \\
D^{\mathrm{III}}(\zeta)=\frac{D_{\mathrm{A}, \text { imp }}^{\mathrm{III}}+D_{\mathrm{B}, \text { self }}^{\mathrm{III}}}{2}-\frac{D_{\mathrm{A}, \text { imp }}^{\mathrm{IIII}}-D_{\mathrm{B}, \text { self }}^{\mathrm{III}}}{2} \operatorname{erf}\left(f\left(C^{\mathrm{III}}\right)\right)
\end{array}\right.
$$

\section{4) Ternary system interdiffusion}

For a ternary system interdiffusion problem between materials composed of an arbitrary concentration rate, for example, a material A composed of $C_{\mathrm{A}}^{\mathrm{I}}=\mu_{\mathrm{A}}^{\mathrm{I}}$, $C_{\mathrm{A}}^{\mathrm{II}}=\mu_{\mathrm{A}}^{\mathrm{II}}$ and $C_{\mathrm{A}}^{\mathrm{III}}=\mu_{\mathrm{A}}^{\mathrm{III}}$ and a material B composed of $C_{\mathrm{B}}^{\mathrm{I}}=\mu_{\mathrm{B}}^{\mathrm{I}}, C_{\mathrm{B}}^{\mathrm{II}}=\mu_{\mathrm{B}}^{\mathrm{II}}$ and $C_{\mathrm{B}}^{\text {III }}=\mu_{\mathrm{B}}^{\text {III }}$, the solutions are possible under the condition of $\mu_{\mathrm{A}}^{\mathrm{I}}+\mu_{\mathrm{A}}^{\mathrm{II}}+\mu_{\mathrm{A}}^{\mathrm{III}}$ $=1$ and $\mu_{\mathrm{B}}^{\mathrm{I}}+\mu_{\mathrm{B}}^{\mathrm{II}}+\mu_{\mathrm{B}}^{\mathrm{III}}=1$. In that case, we must determine the initial and/or boundary values of diffusivities for each element in the materials A and B. In Equation (20), we first determine the point of $\zeta=\zeta_{\mathrm{A}}$ satisfying a given $C_{\mathrm{A}}^{\mathrm{III}}=\mu_{\mathrm{A}}^{\mathrm{III}}$ 
as follows

$$
\zeta_{\mathrm{A}}=2 \sqrt{D_{\text {int }}^{\mathrm{I}}}\left\{\operatorname{erf}^{-1}\left(2 \mu_{\mathrm{A}}^{\mathrm{III}}-1\right)-\beta^{\mathrm{III}}\right\}
$$

By substituting $\zeta=\zeta_{\mathrm{A}}$ into equation (20) and using a parameter $\gamma$ for the given values of $\mu_{\mathrm{A}}^{\mathrm{I}}$ and $\mu_{\mathrm{A}}^{\mathrm{II}}$, the relations of

$$
\mu_{\mathrm{A}}^{\mathrm{I}}=\frac{\gamma}{2}\left(1-\operatorname{erf}\left(\frac{\zeta_{\mathrm{A}}}{2 \sqrt{D_{\mathrm{int}}^{\mathrm{I}}}}+\beta^{\mathrm{I}}\right)\right) \text { and } \mu_{\mathrm{A}}^{\mathrm{II}}=\frac{1-\gamma}{2}\left(1-\operatorname{erf}\left(\frac{\zeta_{\mathrm{A}}}{2 \sqrt{D_{\mathrm{int}}^{\mathrm{II}}}}+\beta^{\mathrm{II}}\right)\right)
$$

are reasonably valid in the diffusion region of the interdiffusion problem mentioned above.

The initial and/or boundary values of diffusivities in the ternary system interdiffusion, which are applicable to the material A composed of the concentration rate $C_{\mathrm{A}}^{\mathrm{I}}=\mu_{\mathrm{A}}^{\mathrm{I}}, C_{\mathrm{A}}^{\mathrm{II}}=\mu_{\mathrm{A}}^{\mathrm{II}}$ and $C_{\mathrm{A}}^{\mathrm{III}}=\mu_{\mathrm{A}}^{\mathrm{III}}$, are thus obtained as

$$
\left\{\begin{array}{l}
D_{\mathrm{A}}^{\mathrm{I}}=\frac{D_{\mathrm{A}, \text { bny }}^{\mathrm{I}}+D_{\mathrm{B}, \text { imp }}^{\mathrm{I}}}{2}-\frac{D_{\mathrm{A}, \text { bny }}^{\mathrm{I}}-D_{\mathrm{B}, \text { imp }}^{\mathrm{I}}}{2} \operatorname{erf}\left(f\left(\mu_{\mathrm{A}}^{\mathrm{I}}\right)\right) \\
D_{\mathrm{A}}^{\mathrm{II}}=\frac{D_{\mathrm{A}, \text { bny }}^{\mathrm{II}}+D_{\mathrm{B}, \text { imp }}^{\mathrm{II}}}{2}-\frac{D_{\mathrm{A}, \text { bny }}^{\mathrm{II}}-D_{\mathrm{B}, \text { imp }}^{\mathrm{II}}}{2} \operatorname{erf}\left(f\left(\mu_{\mathrm{A}}^{\mathrm{II}}\right)\right) \\
D_{\mathrm{A}}^{\mathrm{III}}=\frac{D_{\mathrm{A}, \text { imp }}^{\mathrm{III}}+D_{\mathrm{B}, \text { self }}^{\mathrm{III}}}{2}-\frac{D_{\mathrm{A}, \text { imp }}^{\mathrm{III}}-D_{\mathrm{B}, \text { self }}^{\mathrm{III}}}{2} \operatorname{erf}\left(f\left(\mu_{\mathrm{A}}^{\mathrm{III}}\right)\right)
\end{array}\right.
$$

by using Equation (21).

In a similar manner, we can determine the initial and/or boundary values of diffusivities $D_{\mathrm{B}}^{\mathrm{I}}, D_{\mathrm{B}}^{\mathrm{II}}$ and $D_{\mathrm{B}}^{\mathrm{III}}$ which are applicable to the ternary system interdiffusion in the material $\mathrm{B}$ composed of the concentration rate $C_{\mathrm{B}}^{\mathrm{I}}=\mu_{\mathrm{B}}^{\mathrm{I}}$, $C_{\mathrm{B}}^{\mathrm{II}}=\mu_{\mathrm{B}}^{\mathrm{II}}$ and $C_{\mathrm{B}}^{\mathrm{III}}=\mu_{\mathrm{B}}^{\mathrm{III}}$. Using these initial and/or boundary values, the analytical solutions of the ternary system interdiffusion are reasonably obtained as

$$
D^{j}(\zeta)=\frac{D_{\mathrm{A}}^{j}+D_{\mathrm{B}}^{j}}{2}-\frac{D_{\mathrm{A}}^{j}-D_{\mathrm{B}}^{j}}{2} \operatorname{erf}\left(\frac{\zeta}{2 \sqrt{D_{\mathrm{int}}^{j}}}+\alpha^{j}\right) \text { for } j=\mathrm{I} \text {, II and III }
$$

and

$$
C^{j}(\zeta)=\frac{\mu_{\mathrm{A}}^{j}+\mu_{\mathrm{B}}^{j}}{2}-\frac{\mu_{\mathrm{A}}^{j}-\mu_{\mathrm{B}}^{j}}{2} \operatorname{erf}\left(\frac{\zeta}{2 \sqrt{D_{\mathrm{int}}^{j}}}+\beta^{j}\right) \text { for } j=\mathrm{I} \text {, II and III }
$$

In the above theory, the $\mathrm{K}$ effect is obtained as

$$
\Delta x_{\text {eff }}=\left|\sum_{j=\mathrm{I}}^{\mathrm{III}} \sqrt{D_{\omega}^{j}}\left(C_{\mathrm{A}}^{j}-C_{\mathrm{B}}^{j}\right)\right| \sqrt{t},
$$

where $\omega \rightarrow \mathrm{A}$ if $C_{\mathrm{A}}^{j}>C_{\mathrm{B}}^{j}$ and $\omega \rightarrow \mathrm{B}$ if $C_{\mathrm{A}}^{j}<C_{\mathrm{B}}^{j} \quad[18]$.

As can be seen from the above analytical method, the successive calculations yield the solutions of a $(N+1)$ elements system interdiffusion problem by solving an interdiffusion problem between a material composed of $N$ elements and a pure material. 


\section{Analysis of Experimental Results}

Kirkendall found that $\mathrm{Zn}$ atoms diffuse faster than $\mathrm{Cu}$ atoms in the $\mathrm{Zn}-\mathrm{Cu}$ alloy [17] [19] [20]. The molybdenum wire set at a point on the initial interface between the pure copper and the brass ( $\mathrm{Cu}-70 \%, \mathrm{Zn}-30 \%)$ was used as an inert marker then. The diffusion treatment was performed at the temperature $880^{\circ} \mathrm{C}$ during 119 days in the experiment. In the present work, we apply the above theory to the historical experimental results first known as the $\mathrm{K}$ effect. The initial values of concentration in Figure 1 are $C_{\mathrm{A}}^{\mathrm{I}}=C_{\mathrm{A}}^{\mathrm{Zn}}=0, C_{\mathrm{A}}^{\mathrm{II}}=C_{\mathrm{A}}^{\mathrm{Cu}}=1$, $C_{\mathrm{B}}^{\mathrm{I}}=C_{\mathrm{B}}^{\mathrm{Zn}}=0.3$ and $C_{\mathrm{B}}^{\mathrm{II}}=C_{\mathrm{B}}^{\mathrm{Cu}}=0.7$ then. In that case, however, it is difficult to apply the analytical method resulting in Equations ((17a) and (17b)), since the pure $\mathrm{Zn}$ melts at a temperature of $420^{\circ} \mathrm{C}$. We cannot thus understand the selfdiffusivity $D_{\mathrm{B}, \text { self }}^{\mathrm{Zn}}$ in the above theory. On the other hand, the diffusivity of a metal near the temperature of melting point becomes about $10^{-12} \mathrm{~m}^{2} \cdot \mathrm{s}^{-1}$ regardless of its kind [8]. The value of $D_{\mathrm{B}, \mathrm{self}}^{\mathrm{Zn}}$ is determined as follows in the present work.

The relation of $\mathrm{K}$ effect generalized from Equation (18) is used for determining the value of $D_{\mathrm{B}, \text { self }}^{\mathrm{Zn}}$ compared with the empirical relation of $\Delta x_{\text {eff }}=m \sqrt{t}$. The relation of

$$
m=\frac{2}{\mu}\left|\left(\sqrt{D_{\mathrm{A}}^{\mathrm{Zn}}}-\sqrt{D_{\mathrm{B}}^{\mathrm{Zn}}}\right)\left(C_{\mathrm{A}}^{\mathrm{Zn}}-C_{\mathrm{B}}^{\mathrm{Zn}}\right)\right|
$$

is thus valid in the present case [15] [18]. For the diffusion length $\mu \sqrt{D t}$, $\mu=2$ is used as a matter of convenience for Equation (18). Here, the impurity diffusivity of $\mathrm{Zn}$ in the pure $\mathrm{Cu}$ given by $D_{\mathrm{A}, \mathrm{mp}}^{\mathrm{Zn}}=8.3 \times 10^{-14} \mathrm{~m}^{2} \cdot \mathrm{s}^{-1}$ is adopted as a $D_{\mathrm{A}}^{\mathrm{Zn}}$ value in the present study [21]. Substituting the initial values, $C_{\mathrm{A}}^{\mathrm{Zn}}=0$, $C_{\mathrm{B}}^{\mathrm{Zn}}=0.3$, and $D_{\mathrm{A}}^{\mathrm{Zn}}=8.3 \times 10^{-14} \mathrm{~m}^{2} \cdot \mathrm{s}^{-1}$ into Equation (27), the relation of

$$
\sqrt{D_{\mathrm{B}}^{\mathrm{Zn}}}=\sqrt{8.3} \times 10^{-7} \mp \frac{5 \mu \mathrm{m}}{3}
$$

is obtained. For example, the experimental data at $785^{\circ} \mathrm{C}$ indicate $m=1.22 \times 10^{-7} \mathrm{~m} \cdot \mathrm{s}^{-0.5}$. With reference to the numerical behavior of solutions, $D_{\mathrm{B}}^{\mathrm{Zn}}=1.25 \times 10^{-12} \mathrm{~m}^{2} \cdot \mathrm{s}^{-1}$ is thus adopted as a physically reasonable value in the present study. Here, the solution of basic diffusion Equation (7) for the present problem is expressed as

$$
C^{\mathrm{Zn}}(x)=\frac{3}{20}\left\{1+\operatorname{erf}\left(\frac{x}{2 \sqrt{D_{\mathrm{int}}^{\mathrm{Zn}} t}}+\beta^{\mathrm{Zn}}\right)\right\},
$$

using the defined $D_{\mathrm{int}}^{\mathrm{Zn}}$ and $\beta^{\mathrm{zn}}$.

In Figure 2, the black curve denotes the concentration profile of $\mathrm{Zn}$ given by Equation (29) of the fixed coordinate system at the temperature $880^{\circ} \mathrm{C}$ and diffusion time $t=4.284 \times 10^{5} \mathrm{~s}$. In the same figure, the red curve denotes the concentration profile of $\mathrm{Zn}$ at a room temperature after the diffusion treatment and 


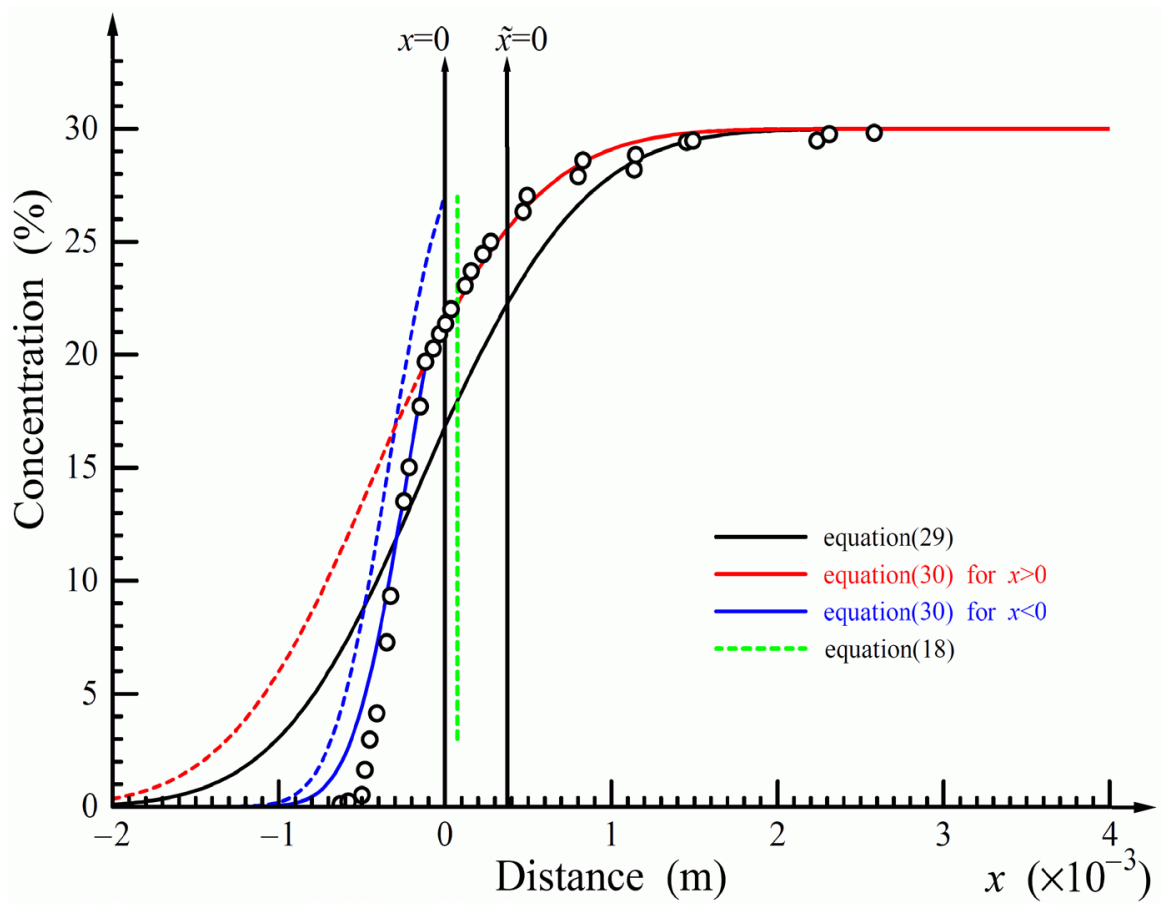

Figure 2. Concentration profile of $\mathrm{Zn}$ in the diffusion region of $\mathrm{Zn}-\mathrm{Cu}$ alloy. The notation $\bigcirc$ indicates an experimental value at a given point in the diffusion region. The black curve shows Equation (29) at the temperature $880^{\circ} \mathrm{C}$ and the time $4.284 \times 10^{5} \mathrm{~s}$ regardless an effect of vacancies. The red curve shows Equation (30) for $x>0$ at a room temperature after the diffusion treatment and it is taken account of an effect of vacancies. The dotted blue curve shows Equation (30) for $x<0$ at a room temperature after the diffusion treatment and the blue curve is taken account of an effect of vacancies in accordance with a situation for $x<0$ then. The dotted green line indicates the so-called Kirkendall effect.

also the $\mathrm{K}$ effect shown by the dotted green line is experimentally obtained as $\Delta x_{\text {eff }}=7.5 \times 10^{-5} \mathrm{~m}$ then. Here, note that the unbalanced vacancies between the regions of $x>0$ and $x<0$ at the temperature $880^{\circ} \mathrm{C}$ and diffusion time $t=4.284 \times 10^{5} \mathrm{~s}$ become a distribution in the thermal equilibrium state at a room temperature.

Considering the inert characteristic of marker, the movement of an inert marker shows that the diffusion region space moves to the inverse orientation against the movement of diffusion particles. As discussed in the previous works, the coordinate transformation of the diffusion equation is thus indispensable for understanding the $\mathrm{K}$ effect [15] [16] [18]. In the present case, therefore, the origin of fixed coordinate system $(t, x)$ is set at the point of mass center on the initial interface and the origin of moving coordinate system $(\tilde{t}, \tilde{x})$ is set at a point of space on the initial interface under the condition of $t=\tilde{t}$ and $x=\tilde{x}+\int_{0}^{t} v \mathrm{~d} \tilde{t}$, where $v$ is a velocity of the origin of $(\tilde{t}, \tilde{x})$ against the origin of $(t, x)$. Equation (27) indicates that the velocity $v$ of the origin of $(\tilde{t}, \tilde{x})$ expressed as

$$
v=\frac{1}{\mu}\left|\left(\sqrt{D_{\mathrm{A}}^{\mathrm{Zn}}}-\sqrt{D_{\mathrm{B}}^{Z n}}\right)\left(C_{\mathrm{A}}^{\mathrm{Zn}}-C_{\mathrm{B}}^{\mathrm{Zn}}\right)\right| t^{-0.5} .
$$


Introducing the effect of shift $x_{\text {sft }}=\int_{0}^{t} v \mathrm{~d} \tilde{t}$ into Equation (29), the concentration profile for $x>0$ at a room temperature is expressed as

$$
C^{\mathrm{Zn}}(x)=\frac{3}{20}\left\{1+\operatorname{erf}\left(\frac{x+\Delta x_{\mathrm{sft}}^{+}}{2 \sqrt{D_{\mathrm{int}}^{\mathrm{Zn}} t}}+\beta^{\mathrm{Zn}}\right)\right\}
$$

using the notation of $\Delta x_{\mathrm{stt}}^{+}=x_{\mathrm{stt}}-\Delta x_{\mathrm{eff}}$, since the excess vacancies disappear during a temperature fall after the diffusion treatment. In other words, the $\tilde{x}=0$ axis and the inert marker on the $x=x_{\text {sft }}$ interface return to $x=\Delta x_{\text {eff }}$ called the Kirkendall interface after the diffusion treatment [15] [16].

The red curve approximately agrees with the experimental results for $x>0$ under the condition of $\Delta x_{\text {stt }}^{+}=3 \times 10^{-4} \mathrm{~m}$, but the dotted red curve is over shifted in the region of $x<0$ compared the experimental results. Resulting from the diffusion behavior of $\mathrm{Zn}$ atoms, the material $\mathrm{B}$ side of diffusion couple is in a supersaturated state of vacancies, while the material A side is in an unsaturated state of vacancies. The red curve of Equation (30) should be thus accepted only in the region for $x>0$. In the present case, the material $\mathrm{A}$ is the pure $\mathrm{Cu}$ and it seems that $\mathrm{Zn}$ atoms on the interface near $x=x_{\mathrm{A}}$ in the diffusion region of $x_{\mathrm{A}} \leq x \leq x_{\mathrm{B}}$ diffuse into the pure $\mathrm{Cu}$ as impurities, if we neglect the vacancy behavior in the diffusion field. This indicates that we can thus adopt $D_{\mathrm{int}}^{\mathrm{Zn}}=D_{\mathrm{A}, \mathrm{imp}}^{\mathrm{Zn}}$ in Equation (30) for $x<0$ as a first approximation.

The dotted blue curve denotes the concentration of $\mathrm{Zn}$ for $x<0$ at a room temperature. Further, taking account of the effect of vacancies for $x<0$, the blue curve shifts to the positive direction of the $x$ axis under the condition of the shift $\Delta x_{\text {stt }}^{-}=\Delta x^{+}-1.2 \times 10^{-4} \mathrm{~m}$ resulting from the unsaturated vacancies for $x<0$. The shifted blue curve agrees approximately with the experimental results for $x<0$ at the room temperature after the diffusion treatment. As a result, this gives an evidence for the validity of present method for determining an initial and/or boundary value of diffusivity discussed in the section 2 .

Using Equations ((29), (30)), the concentration profile of experimental results was physically reproduced as shown by the red curve for $x>0$ and the blue curve for $x<0$ in Figure 2. However, the correlation between $\Delta x_{\text {eff }}$ and $x_{\text {sft }}$ is considerably complicated as discussed in the following. As mentioned above, the diffusion region space moves to the positive direction of $x$ axis in the present case. The inert maker on the initial interface moves to $x=x_{\text {sft }}$ during diffusion treatment in accordance with the movement of $\tilde{x}=0$ axis then. During the temperature fall from $880^{\circ} \mathrm{C}$ to a room temperature after diffusion treatment, the supersaturated vacancies for $x>0$ flow to the negative direction of $x$ axis because of the unsaturated state of vacancies in the region of $x<0$, and at the same time they also flow to the specimen surface considered to be the sink and source of vacancies then. The inert marker does not move to the direction of $x$ axis even if vacancies flow to the specimen surface perpendicular to the $x$ axis. Therefore, the inert marker on the $x=x_{\text {sft }}$ interface returns to $x=\Delta x_{\text {eff }}$ during the temperature fall because of the vacancy flow along the $x$ axis. 


\section{Discussion and Conclusions}

Using the analytical solutions expressed by Equations ((29) and (30)), the interdiffusion problem between the pure copper and brass alloy was reasonably solved in the present study, regardless of the Darken equation. It was concretely confirmed that the Darken equation, which has been widely used for analyzing problems of binary system interdiffusion, is not only actually unnecessary but also theoretically unsuitable for analyzing the interdiffusion problems. By applying the diffusion theory obtained previously to the actual interdiffusion problem, the necessity of coordinate transformation for the diffusion equation was concretely confirmed in the present work. At the same time, it was also confirmed that the concept of intrinsic diffusion is nonexistent from the beginning.

The quantity of excess vacancies corresponding to $\left(x_{\text {sft }}-\Delta x_{\text {eff }}\right)$ returns to the negative direction of $x$ axis after the diffusion treatment. In other words, the quantity of excess vacancies corresponding to $\Delta x_{\text {eff }}$ flows toward the specimen surface. The $\mathrm{K}$ effect thus corresponds to the quantity of vacancies absorbed by the specimen surface considered to be the sink and source of vacancies.

In the region of pure copper, it is considered that diffusion behavior is approximately acceptable as an impurity diffusion mechanism if we neglect the effect of vacancies for interdiffusion problems. In fact, the result based on the concept was reasonably obtained. This gives evidence that the analytical method for solving interdiffusion problems of many elements system is valid, since we can determine initial and/or boundary values of diffusivities as discussed in the Section 2.

For the developments of new useful materials, solving interdiffusion problems between materials is fundamentally one of the most important research subjects. The new analytical method discussed here will be widely applicable to analyzing the interdiffusion problems of many elements system. Behavior of vacancies plays extremely important role in the interdiffusion problems. In order to understand further detailed diffusion behavior, solving the diffusion equation of vacancies in the diffusion region will be necessary [15] [18].

Here, the conclusions obtained from the present work are summarized as follows.

1) It was confirmed that the concept of intrinsic diffusion is not only unnecessary but also wrong from a viewpoint of mathematical physics. The Darken equation should not be used for interdiffusion problems.

2) The Gauss divergence theorem indicates that the diffusion flux $J(t, x)$ given by

$$
J(t, x)=-D \partial C / \partial x+J(t)+J_{\text {eq }}
$$

is valid because of $\partial / \partial x\left\{J(t)+J_{\text {eq }}\right\}=0$ [24]. Here, the flux $J(t)$ means a movement of space within the diffusion region and the $\mathrm{K}$ effect gives evidence for its validity. The flux $J_{\text {eq }}$ independent of the time and space plays an important role in the self-diffusion mechanism [15]. It was also confirmed that the 
theoretical relation of $\mathrm{K}$ effect derived from $J(t)$ of each element is reasonably valid.

3) We found that the present analytical theory is essentially indispensable for analyzing actual interdiffusion problems.

In Appendix, we would like to report the errors of equations in Refs. [13] [14] [15] [16] [18] relevant to the present work.

\section{References}

[1] Altman, E.I. and Schwarz, U.D. (2010) Advanced Materials, 22, 2854-2869. https://doi.org/10.1002/adma.200903927

[2] Novikov, D., Fieremans, E., Jensen, J.H. and Helpern, J.A. (2011) Nature Physics, 7, 508-514.

[3] Agarwal, K., Gopalakrishnan, S., Knap, M., Müller, M. and Demler, E. (2015) Physical Review Letters, 114, 160401. https://doi.org/10.1103/PhysRevLett.114.160401

[4] Zaburdaev, V., Denisov, S. and Klafter, J. (2015) Review of Modern Physics, 87, 483. https://doi.org/10.1103/RevModPhys.87.483

[5] Lausch, T., Mayer, D., Schmidt, F., Lutz, E. and Widea, A. (2017) Nature Physics, 13, 137-141.

[6] Darken, L. S. (1948) Transactions of the Metallurgical Society of AIME, 175, 184-201.

[7] Okino, T. (2013) Journal of Modern Physics, 4, 1495-1498. https://doi.org/10.4236/jmp.2013.411180

[8] Shewmon, P. G. (1963) Diffusion in solids, McGraw-Hill.

[9] Iijima, Y., Funayama, K., Kosugi, T. and Fukumichi, K. (1996) Philosophical Magazine Letters, 74, 423-428.

[10] Masumura, R.A., Rath, B.B. and Pande, C.S. (2002) Acta Materialia, 50, 4535-4544. https://doi.org/10.1016/S1359-6454(02)00273-2

[11] Nathaniel, R., Scott, D.B. and Jerome, D. (2017) Physical Review E, 95, 013206. https://doi.org/10.1103/PhysRevE.95.013206

[12] Mehrer, H. (2007) Diffusion in Solid. Springer-Verlag Berlin Heidelberg. https://doi.org/10.1007/978-3-540-71488-0

[13] Okino, T. (3011) Materials Transactins, 52, 2220-2227.

[14] Okino, T. (2012) Journal of Modern Physics, 3, 255-259. https://doi.org/10.4236/jmp.2012.33034

[15] Okino, T. (2015) Journal of Modern Physics, 6, 2109-2144. https://doi.org/10.4236/jmp.2015.614217

[16] Okino, T., Cho, H. and Yamada, K. (2015) Journal of Modern Physics, 8, 903-918. https://doi.org/10.4236/jmp.2017.86056

[17] Smigelskas, A. D. and Kirkendall, E.O. (1947) Transactions of the Metallurgical Society of AIME, 171, 130-142.

[18] Okino, T. (2014) Applied Physics Research, 6, 1-7. https://doi.org/10.5539/apr.v6n2p1

[19] Kirkendall, E., Thomasson, L. and Upthegrove, C. (1939) Transactions of the Metallurgical Society of AIME, 133, 186-203.

[20] Kirkendall, E. (1942) Transactions of the Metallurgical Society of AIME, 147, 
104-110.

[21] Hino, J., Tomizuka, C.T. and Wert, C.A. (1957) Acta Metallurgy, 5, 41. https://doi.org/10.1016/0001-6160(57)90153-0

[22] Boltzmann, L. (1894) Annual Review of Physical Chemistry, 53, 959-964. https://doi.org/10.1002/andp.18942891315

[23] Matano, C. (1933) Japanese Journal of Physics, 8, 109-113.

[24] Okino, T. (2012) Journal of Modern Physics, 3, 1388-1393. https://doi.org/10.4236/jmp.2012.310175

\section{Appendix}

1) The equation of

$$
\xi_{\mathrm{IN}}=2 \sqrt{D_{\mathrm{A}}^{j} D_{\mathrm{B}}^{j}}\left(D_{\mathrm{A}}^{j}-D_{\mathrm{B}}^{j}\right) /\left(D_{\mathrm{A}}^{j}+D_{\mathrm{B}}^{j}\right) \text { for } j=\mathrm{I} \text {, II and/or no-suffix } j
$$

used at the pages 2223 and 2227 in Ref. [6], at the page 258 in Ref. [7], at the page 2129 in Ref. [8], at the page 911 in Ref. [9] and at the page 5 in Ref. [11] should be revised as

$$
\xi_{\mathrm{IN}}=2 \sqrt{D_{\text {int- }}^{j}}\left(D_{\mathrm{A}}^{j}-D_{\mathrm{B}}^{j}\right) /\left(D_{\mathrm{A}}^{j}+D_{\mathrm{B}}^{j}\right) \text { for } D_{\text {int- }}^{j}=\sqrt{D_{\mathrm{A}}^{j} D_{\mathrm{B}}^{j}}
$$

2) The equation of

$$
\frac{\mathrm{d} C}{\mathrm{~d} \xi_{j}}=\partial_{j}\left(\xi_{n}\right)+\frac{\partial C}{\partial D} \partial_{j} D
$$

used as Equation (4)-(10) at the page 2124 in Ref. [8] should be revised as

$$
\frac{\mathrm{d} C}{\mathrm{~d} \xi_{j}}=\partial_{j} C+\frac{\partial C}{\partial D} \partial_{j} D \text { for } C=C\left(\xi_{n}\right)
$$

3) The equation of

$$
\beta^{j}=-\frac{\xi_{\mathrm{IN}}}{2 \sqrt{D_{\text {int- }}^{j}}}+\operatorname{erf}^{-1}\left\{\frac{C_{\mathrm{A}}^{j}-C_{\mathrm{B}}^{j}}{2}\left(\frac{D_{\mathrm{A}}^{j}+D_{\mathrm{B}}^{j}}{D_{\mathrm{A}}^{j}-D_{\mathrm{B}}^{j}}\right)-\frac{2}{\ln D_{\mathrm{A}}^{j}-\ln D_{\mathrm{B}}^{j}}\right\}
$$

used at the page 911 in Ref. [9] should be revised as

$$
\beta^{j}=-\frac{\xi_{\mathrm{IN}}}{2 \sqrt{D_{\text {int- }}^{j}}}+\operatorname{erf}^{-1}\left\{\frac{D_{\mathrm{A}}^{j}+D_{\mathrm{B}}^{j}}{D_{\mathrm{A}}^{j}-D_{\mathrm{B}}^{j}}-\frac{2}{\ln D_{\mathrm{A}}^{j}-\ln D_{\mathrm{B}}^{j}}\right\} .
$$

